\title{
Noise Attenuation Over Additive Gaussian Channels
}

\author{
Dapeng Li and Naira Hovakimyan
}

\begin{abstract}
We address the noise attenuation problem for linear time-invariant (LTI) systems in the presence of Gaussian communication channels. A novel Linear Matrix Inequality (LMI) approach is formulated to solve the problem. A simulation example is discussed to verify the proposed solution.
\end{abstract}

\section{INTRODUCTION}

Recent progress in communication technologies and their use in feedback control systems motivate to look deeper into the interplay of control and communication in a closed-loop feedback architecture. Among several research directions on this topic, a great deal of attention has been given to the case when the communication channels can be modeled as additive white gaussian noise (AWGN) channels [1]. The issue of time delay has been addressed in [2], the encoder/decoder design has been addressed in [3], and the disturbance rejection has been studied in [4].

In this paper we propose a new control design strategy to address the stabilization and the noise attenuation problems in AWGN channels. The design approach is based on linear matrix inequalities (LMI). The LMI solution gives more computational efficiency and it also avails a possibility of dealing with multiple-input-multiple-output (MIMO) channels.

The paper is organized as follows. In Section II, the problem is formulated, and an LMI solution is provided. In Section III the method is extended to a MIMO channel, and in Section IV a numerical example is given to illustrate the proposed algorithm. The paper is concluded in Section V.

Notation:

- The $\mathcal{H}_{2}$ norm of a transfer function matrix $G(s)$, denoted by $\|G\|_{\mathcal{H}_{2}}$, is obtained by $\|G\|_{\mathcal{H}_{2}}=$ $\frac{1}{2 \pi} \int_{-\infty}^{\infty} \operatorname{Trace}\left(G(j \omega) G^{*}(j \omega)\right)$, where $(\cdot)^{*}$ represents the conjugate transpose.

- The $\mathcal{H}_{\infty}$ norm of a transfer function matrix $G(s)$, denoted by $\|G\|_{\mathcal{H}_{\infty}}$, is obtained by $\|G\|_{\mathcal{H}_{\infty}}=$ $\sup _{\omega} \bar{\sigma}\|G(j \omega)\|$, where $\bar{\sigma}(\cdot)$ gives the maximum singular value.

- The expectation operator is denoted by $\mathbf{E}(\cdot)$.

- The power spectral density (PSD) of a wide-sense stationary signal $x(t), t \geq 0$ is denoted by $f_{e}(\omega)$. If $e(t)$ is an $n$ dimensional vector, then $f_{e}(\omega)$ is matrix.

Research is supported by AFOSR under Grant FA9550-09-1-0265.

D. Li and N. Hovakimyan are with Department of Mechanical Science and Engineering, UIUC, Urbana, IL 61801, e-mail:\{li63, nhovakim\}@illnois.edu

\section{Single InPut Single Output Channel}

We consider the problem of stabilizing an unstable plant over a noisy communication channel, while keeping a certain performance bound for the channel noise attenuation. We consider the following system:

$$
\begin{aligned}
\dot{x}(t) & =A x(t)+B u(t) \quad x(0)=x_{0} . \\
z(t) & =C x(t)
\end{aligned}
$$

where state $x(t) \in \mathbb{R}^{n}$, control input $u(t) \in \mathbb{R}$, performance output $z(t)$. We also assume the system is initialized with a zero-mean Gaussian random variable $x_{0}$. Here we assume that $(A, B, C)$ is a minimal realization. The closed loop is shown in Fig. 1.

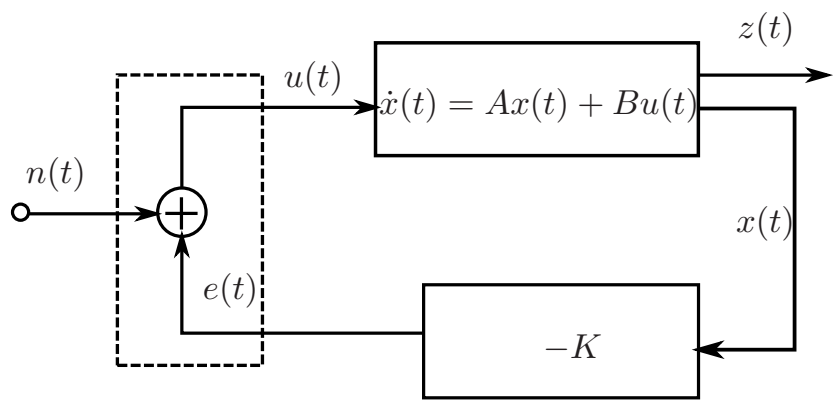

Fig. 1: Closed-loop system

The communication channel is assumed to be an infinite bandwidth AWGN channel as follows

$$
u(t)=e(t)+n(t), \quad t \geq 0,
$$

where $e(t)=-K x(t)$ is the channel input and $K \in \mathbb{R}^{1 \times n}$ is the control gain matrix, $u(t)$ is the channel output, and $n(t)$ is a zero-mean white Gaussian process with PSD $\sigma_{n}^{2}$. The power of the channel input signal is given by $\mathbf{E}\left(e^{2}(t)\right)$, which can be alternatively expressed as

$$
E e^{2}(t)=\frac{1}{2 \pi} \int_{-\infty}^{\infty} f_{e}(\omega) d \omega .
$$

A power constraint is imposed on the input of the AWGN channel as $\mathbf{E} e^{2}(t) \leq \mathcal{P}, \forall t \geq 0$, where $\mathcal{P}>0$ is a prespecified value, reflecting the hardware limitations or some other design requirements. We define the following Signalto-Noise Ratio, or SNR of the channel (2) as

$$
\mathrm{SNR} \triangleq \frac{\mathcal{P}}{\sigma_{n}^{2}} \text {. }
$$


It has been shown that the channel capacity is $\mathrm{SNR} / 2$ nat/sec [5].

Three important aspects of the closed-loop system are considered and analyzed in detail.

1) Closed loop stability: The closed loop system is stabilized by choosing the control gain $K$ from the admissible set $\mathcal{K} \triangleq\{K: A-B K$ is Hurwitz $\}$.

2) Power Constraint: Denote the transfer function from $n(t)$ to the channel input $e(t)$, also known as complementary sensitivity, as $T_{e n}(s)$. The following relation holds [6]:

$$
\frac{1}{2 \pi} \int_{-\infty}^{\infty} f_{e}(\omega) d \omega=\left\|T_{e n}\right\|_{\mathcal{H}_{2}}^{2} f_{n}(\omega)=\left\|T_{e n}\right\|_{\mathcal{H}_{2}}^{2} \sigma_{n}^{2} .
$$

Therefore, the power constraint can be equivalently expressed as

$$
\left\|T_{\text {en }}\right\|_{\mathcal{H}_{2}}^{2} \leq \frac{\mathcal{P}}{\sigma_{n}^{2}}=\text { SNR }
$$

3) Channel Noise Attenuation: We are also interested in the impact of the channel noise $n(t)$ on the measurement variable $z(t)$. Consider the closed loop depicted in Fig.1. We say that the channel noise attenuation is achieved with level $\gamma>0$, if

$$
\left\|T_{z n}\right\|_{\mathcal{H}_{\infty}} \leq \gamma
$$

where $T_{z n}(s)$ is the transfer function from $n(t)$ to $z(t)$. Observing the following relation

$$
\left\|T_{z n}\right\|_{\mathcal{H}_{\infty}}=\sup _{\omega \in \mathbb{R}}\left|T_{z n}(j \omega)\right|=\sup _{\omega \in \mathbb{R}} \sqrt{\frac{f_{z}(\omega)}{f_{n}(\omega)}},
$$

the quantity $\left\|T_{n z}\right\|_{\mathcal{H}_{\infty}}$ reflects the the maximum magnitude of $f_{z}(\omega) / \sigma_{n}^{2}$ over all frequencies. We note that the $\mathcal{H}_{\infty}$ norm is not induced by $\mathcal{L}_{2}$ norms of $z(t)$ and $n(t)$ in the conventional sense [7].

We address the following control problem: find a static state feedback control gain $K \in \mathcal{K}$, such that the required SNR is minimized subject to a desired noise attenuation level $\gamma>0$.

Remark 2.1: State feedback is used for the simplicity of the presentation of the main ideas. More complex cases can be considered in a similar manner.

\section{A. Tradeoff Between Signal-to-Noise Ratio and Channel} Noise Attenuation

1) First Order Case: Consider the following first order unstable dynamics

$$
\begin{aligned}
\dot{x}(t) & =a x(t)+u(t), \\
z(t) & =x(t),
\end{aligned}
$$

where $a>0$ and $u(t)=-k x(t)+n(t) k \geq a$. Let the noise attenuation level be $\gamma>0$. We have the following theorem.

Theorem 2.2: The minimal channel SNR for the system (3) to be stable and satisfying the noise attenuation level $\left\|T_{z n}\right\|_{\mathcal{H}_{\infty}} \leq \gamma$ is given by

$$
\mathrm{SNR} \geq \begin{cases}2 a & \gamma \geq \frac{1}{a} \\ \frac{\gamma}{2}\left(a+\frac{1}{\gamma}\right)^{2} & 0<\gamma<\frac{1}{a}\end{cases}
$$

The corresponding control gain is given as

$$
k^{\star}= \begin{cases}2 a & \gamma \geq \frac{1}{a} \\ a+\frac{1}{\gamma} & 0<\gamma<\frac{1}{a}\end{cases}
$$

Proof: See Appendix.

Remark 2.3: This simple example gives us a chance to understand how much extra SNR (channel capacity) is required to attain a given channel noise attenuation level. As Eqn. (3) suggests, an extra amount of SNR is needed if the attenuation level $\gamma$ is larger than $1 / a$. In view of the the fact that the required channel capacity for closed-loop stability is $a$ [1], the quantity $\max \left\{\frac{\gamma}{4}\left(a+\frac{1}{\gamma}\right)^{2}-a, 0\right\}$ can be regarded as the cost of extra channel capacity to attain the attenuation level $\gamma$.

2) Second Order System: A Case Study: We go one step further and consider the second order system:

$$
\begin{aligned}
& \dot{x}(t)=\left[\begin{array}{ll}
1 & 1 \\
0 & 2
\end{array}\right] x(t)+\left[\begin{array}{l}
0 \\
1
\end{array}\right] u(t), \\
& z(t)=\left[\begin{array}{ll}
1 & 0
\end{array}\right] x(t) .
\end{aligned}
$$

The control gain is given by the matrix $K=\left[\begin{array}{ll}k_{1} & k_{2}\end{array}\right] \in$ $\mathbb{R}^{2}$. The AWGN is given as

$$
u(t)=-K x(t)+n(t) .
$$

The following proposition gives the explicit expression of $\left\|T_{z n}\right\|_{\mathcal{H}_{\infty}}$ in terms of $k_{1}$ and $k_{2}$.

Proposition 2.4: The $\left\|T_{z n}\right\|_{\mathcal{H}_{\infty}}$ for the closed-loop system composed of (6), the controller $K$ and the AWGN is given as

$$
\left\|T_{z n}\right\|_{\mathcal{H}_{\infty}}= \begin{cases}\frac{1}{2+k_{1}-k_{2}}{ }_{2} & \left(k_{1}, k_{2}\right) \in \mathcal{S} \\ \frac{\left(k_{2}-3\right) \sqrt{4 k_{1}-\left(k_{2}-1\right)^{2}}}{\left(k_{1}, k_{2}\right) \in \mathcal{T}}\end{cases}
$$

where

$$
\begin{gathered}
\mathcal{S}=\left\{k_{1}=3, k_{2}=3\right\} \cup\left\{\left(3<k_{1}<5\right) \cap\left(3<k_{2}<k_{1}\right)\right\} \\
\bigcup\left\{\left(k_{1} \geq 5\right) \cap\left(3<k_{2}<2+\sqrt{-1+2 k_{1}}\right)\right\} \\
\mathcal{T}=\left\{k_{1}>5\right\} \bigcap\left\{2+\sqrt{-1+2 k_{1}}<k_{2} \leq k_{1}\right\} \\
\text { Proof: See Appendix. }
\end{gathered}
$$

Now we proceed to calculate the power of channel input $e$ as summarized by the following proposition.

Proposition 2.5: The power of the channel input, in terms of $k_{1}$ and $k_{2}$ can be written as

$$
\frac{1}{2 \pi} \int_{-\infty}^{\infty} f_{e}(\omega) d \omega=\frac{k_{2}^{3}-\left(k_{1}+3\right) k_{2}^{2}+2 k_{1} k_{2}-k_{1}^{2}}{2\left(-3+k_{2}\right)\left(-2-k_{1}+k_{2}\right)} .
$$

Proof: See Appendix.

Unlike the first order case, the increased degree of complexity in the second order case makes it very difficult to get an explicit solution for the problem, even though we have obtained the expressions of the corresponding $\mathcal{H}_{2}$ and $\mathcal{H}_{\infty}$ norms in (9) and (8) respectively. As an alternative, we illustrate the SNR (Channel capacity)/ performance tradeoff graphically in the following plots. 


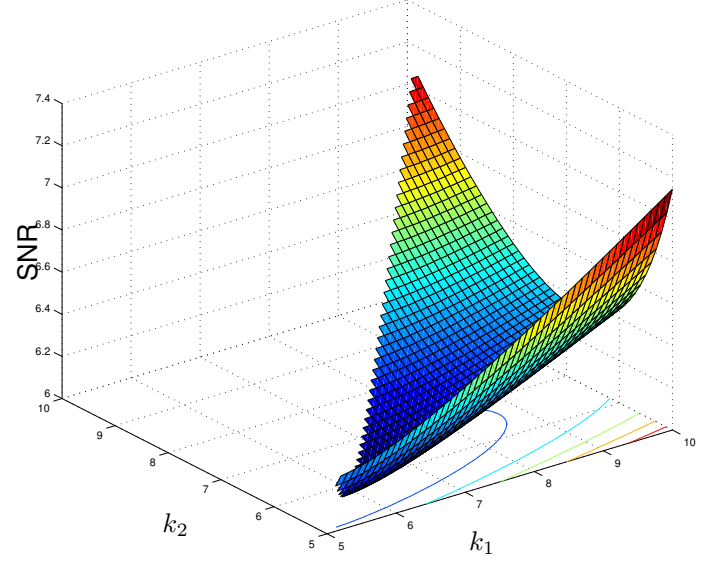

Fig. 2: SNR v.s. Control gain

Fig. 2 shows the required SNR for the given control gain that satisfies the conditions given in (19). As we can see, without an additional constraint for noise attenuation, the minimal SNR is obtained by using the control gain taking the value $\left(k_{1}^{\star}=6, k_{2}^{\star}=6\right)$.

In Fig. 3, the effect of the enforced noise attenuation on the solution set of $K$ is shown. The size of the feasibility set of $K$ decreases along with $\gamma$, which is consistent with (5) for the the first order case.

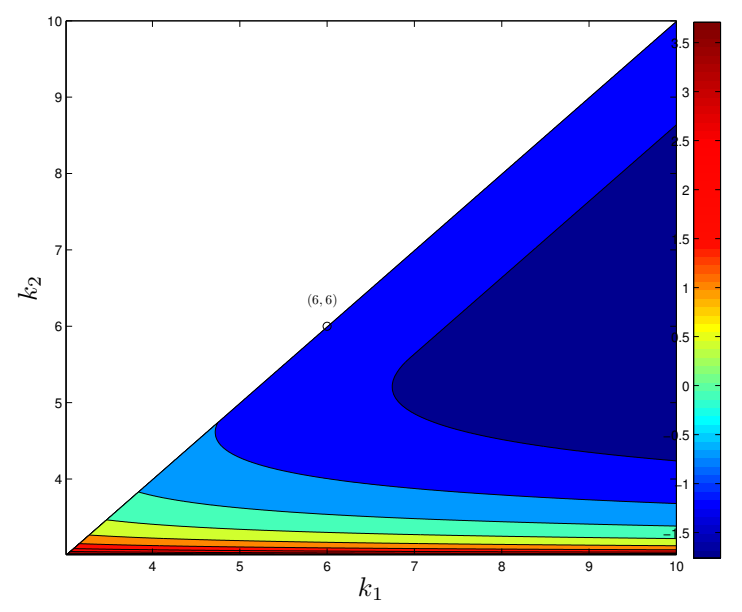

Fig. 3: Feasibility sets for different $\log \gamma$.

\section{B. Controller Design via Linear Matrix Inequality}

In this section, we use LMI technique to solve the problem for the general case. To start with, we introduce the following lemma for SNR minimization.

Lemma 2.6: Consider the closed loop shown in Fig. 1. The optimization problem

$$
\inf _{K \in \mathcal{K}} \operatorname{SNR}
$$

is equivalent to the following LMI minimization problem

$$
\begin{aligned}
& \min _{X_{\mathrm{SNR}}, Y_{\mathrm{SNR}}, \rho} \rho \\
& \text { subject to } \quad X_{\mathrm{SNR}}>0, \Phi\left(X_{\mathrm{SNR}}, Y_{\mathrm{SNR}}, \rho\right) \geq 0 \text { and } \\
& \qquad \Psi\left(X_{\mathrm{SNR}}, Y_{\mathrm{SNR}}\right) \leq 0,
\end{aligned}
$$

where $\rho \in \mathbb{R}, X_{\mathrm{SNR}}=X_{\mathrm{SNR}}^{\top} \in \mathbb{R}^{n \times n}, Y_{\mathrm{SNR}} \in \mathbb{R}^{1 \times n}$,

$$
\Phi\left(X_{\mathrm{SNR}}, Y_{\mathrm{SNR}}, \rho\right) \triangleq\left[\begin{array}{cc}
\rho & Y_{\mathrm{SNR}} \\
Y_{\mathrm{SNR}}^{\top} & X_{\mathrm{SNR}}
\end{array}\right],
$$

and

$$
\Psi\left(X_{\mathrm{SNR}}, Y_{\mathrm{SNR}}\right) \triangleq\left[\left(\begin{array}{cc}
A X_{\mathrm{SNR}}+X_{\mathrm{SNR}} A^{\top} \\
-B Y_{\mathrm{SNR}}-Y_{\mathrm{SNR}}^{\top} B^{\top}
\end{array}\right) X_{\mathrm{SNR}} B\right] .
$$

The optimal control gain is obtained as

$$
K_{\mathrm{SNR}}^{\star}=Y_{\mathrm{SNR}}^{\star}\left(X_{\mathrm{SNR}}^{\star}\right)^{-1},
$$

where $X_{\mathrm{SNR}}^{\star}$ and $Y_{\mathrm{SNR}}^{\star}$ are the optimal solutions to the problem (10).

Proof: The proof is based on the classic LMI solution to the state feedback $\mathcal{H}_{2}$ optimization synthesis problem for the following auxiliary deterministic closed loop, composed of the plant $G(s)$ and the controller $K(s)$ :

$$
G(s)=\left[\begin{array}{c|c}
A & B \\
\hline 0 & 1 \\
\mathbb{I} & 0
\end{array}\right], \quad K(s)=K \in \mathbb{R}^{1 \times n},
$$

with the objective function given by $\| K(s \mathbb{I}-A+$ $B K)^{-1} B \|_{\mathcal{H}_{2}}^{2}$. The proof is completed by using the standard procedure given in [8].

Similarly, the noise attenuation can also be cast into LMI conditions, given by the following lemma.

Lemma 2.7: Consider the closed loop in Fig. 1. The noise attenuation level is less than $\gamma$, if and only if there exist matrices $0<X_{n}=X_{n}^{\top} \in \mathbb{R}^{n \times n}, Y_{n} \in \mathbb{R}^{1 \times n}$ that satisfy the following LMI feasibility condition

$$
\Theta_{\gamma}\left(Y_{n}, X_{n}\right) \triangleq\left[\begin{array}{ccc}
\left(\begin{array}{c}
A X_{n}+X_{n} A^{\top} \\
-B Y_{n}-Y_{n}^{\top} B^{\top}
\end{array}\right) & B & X_{n} C^{\top} \\
B^{\top} & -1 & 0 \\
C X_{n} & 0 & -\gamma^{2}
\end{array}\right]
$$

The resulting control gain is obtained as

$$
K_{n}=Y_{n} X_{n}^{-1} \text {. }
$$

Proof: Consider the following auxiliary system $G$ and the controller $K$ respectively

$$
G(s)=\left[\begin{array}{c|cc}
A & B & B \\
\hline C & 0 & 0 \\
\mathbb{I} & 0 & 0
\end{array}\right], \quad K(s)=K_{n} \in \mathbb{R}^{1 \times n} .
$$

The transfer function from the disturbance to the performance measurement is calculated as

$$
S(s)=C\left(s \mathbb{I}-A+B K_{n}\right)^{-1} B,
$$

which is identical to $T_{z n}(s)$. Subsequently we can use standard LMI arguments to obtain the feasibility sets of $X_{n}$ 
and $Y_{n}$ that satisfy $\left\|T_{z n}(s)\right\|_{\mathcal{H}_{\infty}} \leq \gamma$. The readers can refer to [8] for details.

It is easy to see that the problem proposed in Section II is equivalent to minimizing $\rho$ over all matrices $X_{\mathrm{SNR}}, Y_{\mathrm{SNR}}, X_{n}, Y_{n}, \rho$ that satisfy (10) and (11). While the optimization problems in (10) and (11) are convex themselves, the joint one is not convex. Therefore we enforce the condition

$$
X=X_{\mathrm{SNR}}=X_{n} \text { and } Y=Y_{\mathrm{SNR}}=Y_{n}
$$

to obtain the convexity, admittedly with some degree of conservatism. Indeed, the same treatment is widely used in mixed $\mathcal{H}_{2} / \mathcal{H}_{\infty}$ problems, such as [9]. The above argument proves the following main theorem.

Theorem 2.8: Given a desired channel noise attenuation level $\gamma$, a lower bound for the required channel SNR of the closed loop system is obtained via the following LMI optimization problem:

$$
\begin{array}{ll}
\min _{X, Y} & \rho \\
\text { Subject to } & X>0, \Phi(X, Y, \rho)>0 \\
& \Psi(X, Y) \leq 0 \text { and } \Theta_{\gamma}(X, Y) \leq 0
\end{array}
$$

The lower bound of the SNR is given as $\rho^{\star}$, which is the optimal value obtained in (12). The corresponding controller is given as

$$
K^{\star}=Y^{\star}\left(X^{\star}\right)^{-1},
$$

where $X^{\star}, Y^{\star}$ are the resulting values of the decision variables $X$ and $Y$ respectively.

Remark 2.9: In this section, only the full state feedback is considered. However, the same approach can be easily extended to output feedback case.

\section{Vector Gaussian Channel}

Here we consider the case where the control signal is a vector, and it is transmitted through a vector Gaussian channel, which is also a simple case of a MIMO channel. In applications, this scenario represents the case, where actuators and controllers are geographically distributed and multiple transmitters and receivers are therefore employed to conduct the communication task, as shown in Fig. 4. From the perspective of wireless communication, a multiple access system with multiple antennas at the base-station allows several users, who are spatially separated, to communicate simultaneously. Moreover, the channel fading in the pointto-point communication can be overcome or even utilized by MIMO communication schemes [10].

The channel is modeled as follows:

$$
u(t)=H e(t)+n(t) \quad t \geq 0,
$$

where $e(t) \in \mathbb{R}^{m}$ is the channel input, and $u(t) \in \mathbb{R}^{m}$ is the channel output, $n(t)$ is a $m$ dimensional gaussian white noise process with $E n(t)=0$ and $E n(t) n^{\top}(t)=\sigma_{n}^{2} \mathbb{I}$, and $H \in \mathbb{R}^{m \times m}$ is a channel gain matrix, which is assumed to be deterministic here. The channel input is required to satisfy the following power constraint as

$$
\mathbf{E} e^{\top}(t) e(t)=\operatorname{Trace}\left(\mathbf{E}\left(e(t) e^{\top}(t)\right)\right) \leq \mathcal{P} \quad \forall t \geq 0
$$

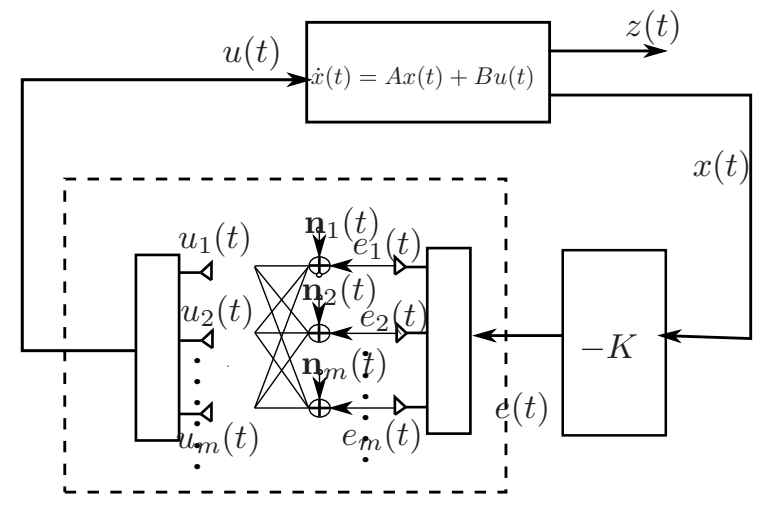

Fig. 4: Control over a Vector Gaussian Channel

for some pre-specified input power level $\mathcal{P}>0$. Similar to the scalar case, the power of the channel input $\mathbf{E} e^{\top}(t) e(t)$ can be also represented as

$$
\operatorname{Trace}\left(\mathbf{E}\left(e(t) e^{\top}(t)\right)\right)=\frac{1}{2 \pi} \int_{-\infty}^{\infty} \operatorname{Trace} f_{e}(j \omega) d \omega .
$$

Here the SNR is similarly defined as

$$
\mathrm{SNR}:=\frac{\mathcal{P}}{\sigma_{n}^{2}} .
$$

\section{A. State Feedback Stabilization}

In this section, we design a controller/transmitter $K$, such that the closed loop system satisfies the power constraint $\mathcal{P}$. We can then formulate the following theorem for the solution of SNR constrained state feedback stabilization.

Lemma 3.1: Consider the feedback configuration in Fig. 4, where we have

$$
\begin{array}{cl}
\min & \operatorname{Trace}(\Omega) \\
\text { subject to } & X_{\mathrm{SNR}}>0, \tilde{\Phi}\left(X_{\mathrm{SNR}}, Y_{\mathrm{SNR}}, \Omega\right)>0 \text { and } \\
& \tilde{\Psi}\left(X_{\mathrm{SNR}}, Y_{\mathrm{SNR}}\right) \leq 0
\end{array}
$$

in which $\Omega \in \mathbb{R}^{m \times m}, X_{\mathrm{SNR}}=X_{\mathrm{SNR}}^{\top} \in \mathbb{R}^{n \times n}, Y_{\mathrm{SNR}} \in \mathbb{R}^{m \times n}$,

$$
\tilde{\Phi}\left(X_{\mathrm{SNR}}, Y_{\mathrm{SNR}}, \Omega\right) \triangleq\left[\begin{array}{cc}
\Omega & Y_{\mathrm{SNR}} \\
Y_{\mathrm{SNR}}^{\top} & X_{\mathrm{SNR}}
\end{array}\right]
$$

and

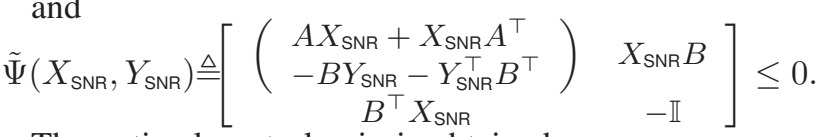

The optimal control gain is obtained as

$$
K_{\mathrm{SNR}}^{\star}=H^{-1} Y_{\mathrm{SNR}}^{\star}\left(X_{\mathrm{SNR}}^{\star}\right)^{-1},
$$

where $Y_{\mathrm{SNR}}^{\star}$ and $X_{\mathrm{SNR}}^{\star}$ are the solutions of the optimization problem.

Proof: Note that the power of the channel input can be represented as

$\operatorname{Trace}\left(\mathbf{E} e(t) e^{\top}(t)\right)=\frac{1}{2 \pi} \int_{-\infty}^{\infty} \operatorname{Trace} f_{e}(j \omega) d \omega$ 


$$
\begin{aligned}
& =\frac{\sigma^{2}}{2 \pi} \int_{-\infty}^{\infty} \operatorname{Trace}\left(T_{n e}(j \omega) T_{n e}^{*}(j \omega)\right) d \omega \\
& =\left\|T_{n e}\right\|_{\mathcal{H}_{2}} \sigma^{2},
\end{aligned}
$$

where the transfer function $T_{n e}(s)$ is written as

$$
T_{n e}(s)=H K(s \mathbb{I}-A+B H K)^{-1} B .
$$

Therefore the problem is reduced to the following $\mathcal{H}_{2}$ optimization problem:

$$
\inf _{K}\left\|T_{n e}\right\|_{\mathcal{H}_{2}}
$$

To minimize the channel SNR, we consider the following auxiliary deterministic closed loop, composed of the plant $G(s)$ and the controller $K(s)$ :

$$
G(s)=\left[\begin{array}{c|c}
A & B \\
\hline 0 & 1 \\
\mathbb{I} & 0
\end{array}\right], \quad K(s)=H K, K \in \mathbb{R}^{m \times n},
$$

for which we minimize the $\mathcal{H}_{2}$ norm of $T_{n e}(s)$. The solution can be obtained by solving the standard $\mathcal{H}_{2}$ optimal control problem via LMIs [8].

Similar to the scalar case, the noise attenuation is also presented via relevant LMI conditions in the following lemma.

Lemma 3.2: Consider the closed loop in Fig. 4. The noise attenuation level is less than $\gamma$, if and only there exist matrices $0<X_{n}=X_{n}^{\top} \in \mathbb{R}^{n \times n}, Y_{n} \in \mathbb{R}^{m \times n}$ that satisfy the following LMI feasibility condition

$$
\begin{aligned}
& \tilde{\Theta}_{\gamma}\left(Y_{n}, X_{n}\right) \triangleq \\
& \quad\left[\begin{array}{ccc}
\left(\begin{array}{c}
A X_{n}+X_{n} A^{\top} \\
-B Y_{n}-Y_{n}^{\top} B^{\top}
\end{array}\right) & B & X_{n} C^{\top} \\
B^{\top} & -\mathbb{I} & 0 \\
C X_{n} & 0 & -\gamma^{2} \mathbb{I}
\end{array}\right] \leq \alpha(17)
\end{aligned}
$$

The resulting control gain is obtained as

$$
K_{n}=H^{-1} Y_{n} X_{n}^{-1}
$$

Then we have the following theorem.

Theorem 3.3: Given the channel noise attenuation level $\gamma$, a lower bound for the required channel SNR of the closed loop system is obtained via the solution of the following LMI optimization problem:

$$
\begin{array}{ll}
\min _{X, Y} & \operatorname{Trace}(\Omega) \\
\text { Subject to } & X>0, \tilde{\Phi}(X, Y, \Omega)>0 \\
& \tilde{\Psi}(X, Y) \leq 0 \text { and } \tilde{\Theta}_{\gamma}(X, Y) \leq 0 .
\end{array}
$$

The (sub)optimal value of $\operatorname{SNR}$ is given as $\operatorname{Trace}\left(\Omega^{\star}\right)$, and the corresponding controller is given as

$$
K^{\star}=Y^{\star}\left(X^{\star}\right)^{-1}
$$

where $X^{\star}, Y^{\star}$ are the optimal values of the decision matrices $X$ and $Y$ respectively.

\section{NUMERICAL EXAMPLE}

In this section we will give a simple example to illustrate

\begin{tabular}{|c|c|c|c|c|}
\hline$\gamma$ & $\min \left\|T_{e n}\right\|_{\mathcal{H}_{2}}^{2}$ & \multicolumn{3}{|c|}{$K$} \\
\hline 0.1 & 22.5095 & $\begin{array}{c}-2.9905 \\
16.5871\end{array}$ & $\begin{array}{l}58.9790 \\
-8.4777\end{array}$ & $\begin{array}{l}19.0237 \\
-3.6414\end{array}$ \\
\hline 0.5 & 16.7534 & $\begin{array}{c}-2.5710 \\
16.4738\end{array}$ & $\begin{array}{c}21.5600 \\
-0.2014\end{array}$ & $\begin{array}{c}9.6628 \\
-1.5698\end{array}$ \\
\hline 1 & 16.2293 & $\begin{array}{c}-2.4630 \\
16.4472\end{array}$ & $\begin{array}{c}17.8123 \\
0.7493\end{array}$ & $\begin{array}{c}8.7206 \\
-1.3309\end{array}$ \\
\hline 10 & 16.0064 & $\begin{array}{c}-2.3994 \\
16.4279\end{array}$ & $\begin{array}{c}15.9983 \\
1.2303\end{array}$ & $\begin{array}{c}8.2658 \\
-1.2101\end{array}$ \\
\hline
\end{tabular}
the proposed algorithm. We consider the following state space realization of a 3rd order LTI system:

$$
\begin{gathered}
A=\left[\begin{array}{lll}
4 & 1 & 0 \\
0 & 2 & 1 \\
0 & 0 & 2
\end{array}\right], \quad B=\left[\begin{array}{cc}
0 & 0.5 \\
0 & 0 \\
1 & 0
\end{array}\right], \\
C=\left[\begin{array}{lll}
0 & 0 & 1
\end{array}\right] .
\end{gathered}
$$

The vector Gaussian channel has two inputs and two outputs, where the gaussian noise vector $n(t) \in \mathbb{R}^{2}$ and $\mathbb{E} n(t)=[0,0]^{\top}, \mathbb{E} n^{\top}(t) n(t)=\mathbb{I}$, and the channel matrix is given by

$$
H=\left[\begin{array}{cc}
1 & 0.2 \\
0.2 & 1
\end{array}\right]
$$

We first calculate the control gains $(K)$ and the minimal channel input power $\left(\left\|T_{e n}(j \omega)\right\|_{\mathcal{H}_{2}}^{2}\right.$, for different values of $\gamma$. The result is summarized in the following table.

Upon obtaining the control gains for different $\gamma$ 's we can compare the corresponding PSDs of the observation signal $z$, which are depicted in Fig. 5.

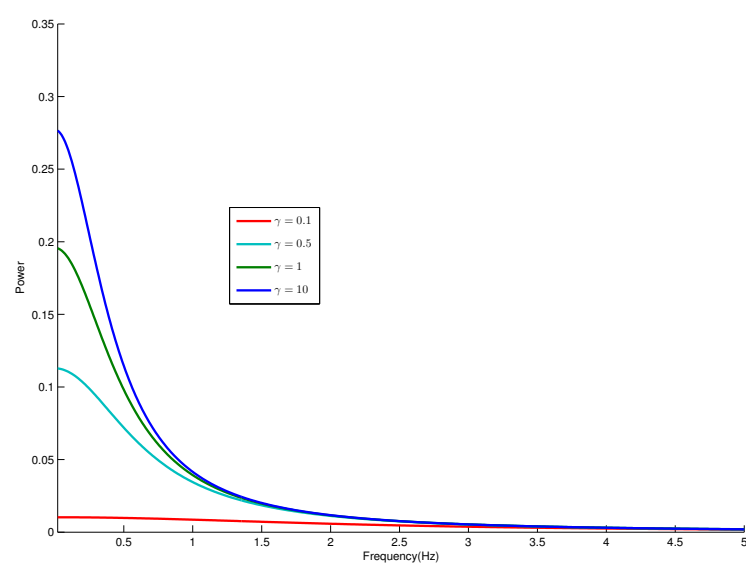

Fig. 5: Power Spectral Density of $z$ for different noise attenuation levels

As we can see from Fig. 5, setting $\gamma$ lower implies that the impact of the channel noise on the observation signal $z$ is smaller. It is also not surprising to see that $\min \left\|T_{n e}\right\|_{\mathcal{H}_{2}}$ tends to its ideal value 16 (twice of the sum of all the unstable eigenvalues of $A$ ) as the noise attenuation requirement is eased. 
Fig. 6 shows the relation between the minimal SNR and the noise attenuation level $\gamma$.

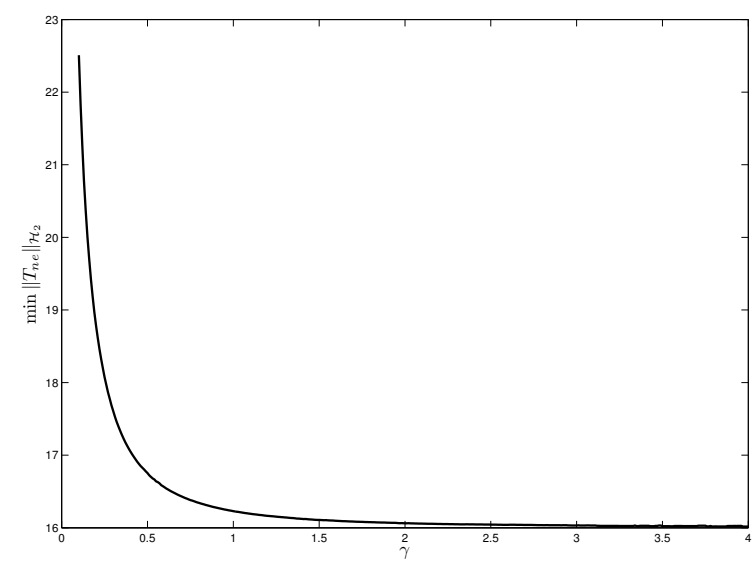

Fig. 6: Minimal SNR (power of $z$ ) versus $\gamma$

\section{CONCLUSION}

In this paper, we have considered the channel noise attenuation problem for feedback control over both scalar and vector gaussian channels. An effective LMI approach is proposed and verified. Future development includes uncertain systems and output feedback.

\section{REFERENCES}

[1] J. Braslavsky, R. Middleton, and J. Freudenberg, "Feedback stabilization over signal-to-noise ratio constrained channels," IEEE Transactions on Automatic Control, vol. 52, no. 8, pp. 1391-1403, 2007.

[2] A. Rojas, J. Braslavsky, and R. Middleton, "Brief paper: Fundamental limitations in control over a communication channel," Automatica, vol. 44, no. 12 , pp. 3147-3151, 2008.

[3] G. Goodwin, D. Quevedo, and E. Silva, "Architectures and coder design for networked control systems," Automatica, vol. 44, no. 1, pp. 248-257, 2008.

[4] A. Rojas, R. Middleton, J. Freudenberg, and J. Braslavsky, "Input disturbance rejection in channel signal-to-noise ratio constrained feedback control," in American Control Conference, 2008, 2008, pp. 31003105 .

[5] S. Ihara, Information theory for continuous systems. World Scientific Pub Co Inc, 1993.

[6] K. Aström, Introduction to stochastic control theory. Academic Press, 1970.

[7] K. Zhou and J. Doyle, Essentials of robust control. Prentice Hall New Jersey, 1998.

[8] S. Boyd, L. Ghaoui, E. Feron, and V. Balakrishnan, Linear matrix inequalities in system and control theory. SIAM Studies in Applied Mathematics, 1994.

[9] M. Chilali and P. Gahinet, " $H_{\infty}$ design with pole placement constraints: an LMI approach," IEEE Transactions on Automatic Control, vol. 41, no. 3, pp. $358-367$, mar 1996.

[10] D. Tse and P. Viswanath, Fundamentals of wireless communication. Cambridge Univ Pr, 2005.

[11] J. Conway, Functions of one complex variable II. Springer, 1995.

\section{APPENDIX}

\section{A. Proof of Theorem 2.2}

Calculate the inequality $\left\|T_{z n}\right\|_{\mathcal{H}_{\infty}} \leq \gamma$ as follows

$$
\gamma \geq \sup _{\omega \in \mathbb{R}}\left|T_{z n}(j \omega)\right|=\sup _{\omega \in \mathbb{R}} \frac{1}{\sqrt{\omega^{2}+(a-k)^{2}}}=\frac{1}{k-a} .
$$

Then we have $k \geq a+1 / \gamma$ as an additional constraint for the minimization of the power of the channel input signal $k x$. This optimization problem is formulated and explicitly solved as

$$
\begin{aligned}
\inf _{k \geq a+1 / \gamma}\|k x\|_{\mathcal{H}_{2}}^{2} & =\inf _{k \geq a+1 / \gamma} k \frac{1}{2 \pi} \int_{-\infty}^{\infty} \frac{\sigma_{n}^{2}}{\omega^{2}+(k-a)^{2}} d \omega \\
& =\inf _{k \geq a+1 / \gamma} \sigma_{n}^{2} \frac{k^{2}}{k-a} \\
& = \begin{cases}2 a \sigma_{n}^{2} \\
\frac{\gamma}{2}\left(a+\frac{1}{\gamma}\right)^{2} \sigma_{n}^{2} & 0<\gamma<\frac{1}{a} .\end{cases}
\end{aligned}
$$

Eqs. (4) and (5) follow straightforwardly.

\section{B. Proof of Proposition 2.4}

We calculate $\|e\|_{\mathcal{H}_{2}}^{2}$ by using the following complex contour integral and the residue theorem [11]:

$$
\begin{aligned}
& \|e\|_{\mathcal{H}_{2}}^{2} \\
& =\frac{\sigma_{n}^{2}}{2 \pi} \int_{-\infty}^{\infty} T_{e n}(j \omega) T_{e n}(-j \omega) d \omega \\
& =\frac{\sigma_{n}^{2}}{2 \pi j} \oint_{\gamma} T_{e n}(s) T_{e n}(-s) d s \\
& =\sigma_{n}^{2}\left(\operatorname{Res}\left(T_{e n}(s) T_{e n}(-s) ; p_{1}\right)+\operatorname{Res}\left(T_{e n}(s) T_{e n}(-s) ; p_{2}\right)\right) \\
& =\sigma_{n}^{2} \frac{k_{2}^{3}-\left(k_{1}+3\right) k_{2}^{2}+2 k_{1} k_{2}-k_{1}^{2}}{2\left(-3+k_{2}\right)\left(-2-k_{1}+k_{2}\right)}
\end{aligned}
$$

where $\gamma$ represents the contour $[-j \omega R, j \omega R] \cup\{R \exp (j \theta)$ : $-\pi / 2<\theta<\pi / 2\}$ with large enough radius $R>0$, and $\operatorname{Res}\left(\cdot ; p_{i}\right)$ denotes the residue evaluated at the poles $p_{i}, i=$ 1,2 . During the course of calculation we have used the fact that

$$
T_{e n}(s)=\frac{k_{2} s+k_{1}-k_{2}}{s^{2}+\left(k_{2}-3\right) s+k_{1}-k_{2}+2} .
$$

The conclusion is therefore reached by noticing that SNR = $\|e\|_{\mathcal{H}_{2}}^{2} / \sigma_{n}^{2}$.

\section{Proof of Proposition 2.5}

First note that

$$
T_{z n}(s)=\frac{1}{s^{2}+\left(k_{2}-3\right) s+k_{1}-k_{2}+2},
$$

and by using Routh's criterion, the set of stabilizing control gains is obtained as

$$
\mathcal{K}=\left\{\left[k_{1} k_{2}\right]:\left\{k_{1} \geq k_{2}\right\} \cap\left\{k_{2} \geq 3\right\}\right\} .
$$

The rest of the proof follows the procedure of solving the optimization problem

$$
k_{1} \text { and } k_{2} \text { satisfy (19) }
$$

$$
\sqrt{T_{z n}(j \omega)_{z n}(-j \omega)}
$$

The machinery used for this problem is reduced to calculus, and is dropped therefore. 